\title{
Survey of antibiotic treatment of Escherichia coli infection in broilers and efficacy of Enrofloxacin plus colistin in experimental Colibacillosis
}

\author{
Tanveer Ahmad ${ }^{1}$, Ghulam Muhammad², Aamir Sharif ${ }^{3 *}$, Muhammad \\ Nadeem ${ }^{4}$, Abdul Shakoor ${ }^{5}$ and Muhammad Rizwan ${ }^{6}$ \\ 1. Department of Clinical Sciences, Faculty of Veterinary Sciences, Bahauddin Zakariya University, Multan- \\ Pakistan \\ 2. Department of Veterinary Clinical Medicine and Surgery, Faculty of Veterinary Sciences, University of \\ Agriculture, Faisalabad-Pakistan \\ 3. Government Poultry Farm, Bahawalpur, Livestock and Dairy Development Department, Punjab, District \\ Bahawalpur-Pakistan \\ 4. Pir Mehr Ali Shah Arid Agriculture University, Rawalpindi, Sub-Campus Khushab-Pakistan \\ 5. College of Veterinary Sciences, Sub- Campus, Jhang, University of Veterinary and Animal Sciences, Lahore- \\ Pakistan \\ 6. College of Veterinary Sciences, Bahadur Campus, Layyah, Bahauddin Zakariya University, Multan-Pakistan \\ *Corresponding author's email: aamirsharifcheema@gmail.com \\ Citation \\ Tanveer Ahmad, Ghulam Muhammad, Aamir Sharif, Muhammad Nadeem, Abdul Shakoor and Muhammad \\ Rizwan. Survey of antibiotic treatment of Escherichia coli infection in broilers and efficacy of Enrofloxacin \\ plus colistin in experimental Colibacillosis. Pure and Applied Biology.Vol. 9, Issue 3, pp1864-1872. \\ http://dx.doi.org/10.19045/bspab.2020.90199
}

\begin{tabular}{llll}
\hline \hline Received: 01/02/2020 & Revised: 25/04/2020 & Accepted: 10/05/2020 & Online First: 19/05/2020 \\
\hline
\end{tabular}

\section{Abstract}

Colibacillosis caused by Escherichia coli is very important disease of poultry and is characterized by development of colisepticemia, pericarditis, perihepatitis, increased mortality and decreased feed conversion rate. Thirty E. coli infected broiler farms located in district Faisalabad were surveyed and data pertaining to antibiotic usage, storage, withdrawal period and farmers perception on antibiotic resistance was obtained. Data showed that most of poultry farmers used antibiotics in combination (Enrofloxacin plus colistin) to treat or control E. coli infection. Morbid samples from suspected E. coli infected birds were collected, pure colonies of were isolated and pathogenicity was determined. In vivo colibacillosis in broilers was induced by oral inoculation of $1 \times 10^{6}$ colony forming units (cfu)/bird and efficacy of enrofloxacin plus colistin was determined. For that purpose one hundred forty (140) broiler birds were divided into four groups (A, B, C and D), each having 35 birds. At $26^{\text {th }}$ day of age $E$. coli infection was induced in all groups. At $28^{\text {th }}$ day of age group A was treated with enrofloxacin plus colistin sulphate, group B with enrofloxacin, group C with colistin sulphate and group D was kept as untreated control. Mortality, cumulative feed consumption, mean body weight, mean weight gain and FCR were recorded in each group. It was found that combined use of enrofloxacin plus colistin in colibacillosis in broilers decreased mortality, increased cumulative feed consumption, increased mean body weight, increased mean weight gain and FCR than single use of either of enrofloxacin or colistin.

Keywords: Antimicrobial therapy; Colibacillosis; Poultry flocks

\section{Introduction}

Colibacillosis caused by $E$. coli is a disease of significant economic concern in all phases of poultry industry in Pakistan. E. coli are worldwide in distribution and are found in intestinal tract of all warm blooded species, also found in the litter and fecal material and persist for long period of time 
under farm conditions. Contaminated feed and drinking water act as a source of colibacillosis. Egg transmission of pathogenic E. coli cause high early chick mortality and morbidity. Prevalence rate of colibacillosis in poultry is $13.44 \%$ [1]. Higher incidence of colibacillosis in poultry is also reported [2-5].

A number of antibiotics are used to treat and prevent bacterial diseases of poultry. Strains of E. coli are generally resistant to a variety of antibiotics used as therapeutic, preventive or growth promoting agents [68]. No single antimicrobial agent may be effective against all $E$. coli isolates in a geographic region [5]. As a result there is a need for conjoined antimicrobial therapy.

Enrofloxacin is carboxylic acid quinolone derivative, used for combating many bacterial diseases. It has a wide antibacterial spectrum against mycoplasma, salmonella, E. coli, Haemophihus paragalinarum, Pasteurella multocida and staphylococcus in chicken [9-11]. Colistin (polymyxin E) is a cationic surface-active agent that disrupts the structure of cell membrane phospholipids and increases cell permeability by a detergent like action. It disorganizes the outer membrane of bacteria by binding to phospholipid (endotoxin) through direct interaction of the cationic drugs with the anionic lipid-A region. Colistin is synergistic with a variety of antimicrobial drugs in its disorganizing effects on the outer and cytoplasmic membrane. It is used in veterinary practice against many bacterial diseases in chicken and is effective against gram-negative bacteria such as E. coli, salmonella, pasteurella and pseudomonas [12]. Although this antibiotic has been commonly prescribed in combination with enrofloxacin. The published report on the efficacy of this combination under Pakistani conditions is lacking. The present study was therefore, designed to investigate the therapeutic efficacy of combination of enrofloxacin and colistin in an experimentally induced colibacillosis in broiler chicks.

\section{Materials and methods \\ Part - I \\ Field Survey of antibiotic usage for treatment of $E$. coli}

Thirty E. coli infected poultry farms located in district Faisalabad were surveyed and following data was obtained.

1. Antibiotic usage (single or combination, name of antibiotic, brand name, manufacturer), pre-treatment, post treatment mortality and percent reduction of mortality.

2. Awareness of antibiotic usage patterns i.e. duration, efficacy, storage, price, brand, with drawl period, etc.

\section{Part - II}

\section{Isolation and identification of $\boldsymbol{E}$. coli}

Livers and hearts of sick and dead birds of different age groups suffering from suspected $E$. coli infection were collected. Samples were transported to the laboratory and inoculated on MacConkey's agar medium for primary isolation, purification and identification of $E$. coli from samples [13].

\section{Morphology and staining reaction}

The morphological, cultural, staining characteristics, of isolates were determined. The smears were prepared and stained with gram's staining and examined under microscope. The morphology and staining reaction of each isolate was recorded. For biochemical and pathgenicity test, the isolates were maintained on MacConkey's agar slants.

\section{Sugar fermentation and Biochemical tests}

Sugar fermentation and biochemical tests including triple sugar iron reaction and citrate utilization test were performed for confirmation of $E$. coli [13-15].

\section{Pathogenicity of $E$. coli}

Gut loop assay was performed for determination of pathogenicity of $E$. coli. The rabbits were anesthetized with Ketamine HCI and the ileum was exposed. The ileum was cut and half milliliter culture of the test isolate was delivered into the lumen of the intestine. Equal volume of normal saline was injected into the intestine 
in control group. The lumen of intestine was sutured with catgut and animals were kept under care. The observations were recorded [16].

\section{Preparation of inoculum}

After confirmation of the pathogenicity, $E$. coli isolate was inoculated into fresh nutrient broth and incubated at $37^{\circ} \mathrm{C}$ for overnight. The viable count of bacteria was carried [13]. The broth was diluted with phosphate buffer saline (PBS) to have approximately $1 \times 10^{6} \mathrm{cfu} / \mathrm{mL}$. The inoculums were further used for experimental infection.

\section{Experimental design}

140 day old broiler chicks (Hubard $\times$ Hubard) were purchased and were reared under uniform conditions of management and nutrition. The birds were fed with broiler starter ration from day 1 to $28^{\text {th }}$ days and broiler finisher ration from $29^{\text {th }}$ to $40^{\text {th }}$ days. All the birds were vaccinated according to the schedule (Table 1).

At $25^{\text {th }}$ day, birds were divided into four groups (A, B, C and D), each having 35 birds. At age of $26^{\text {th }}$ day E. coli infection was induced by oral inoculation of $1 \mathrm{~mL}$ of $1 \times 10^{6} \mathrm{cfu} / \mathrm{bird}$ in all groups. At $28^{\text {th }}$ day of age Group A was treated with Enrofloxacin plus Colistin Sulphate, group B with Enrofloxacin, group C with Colistin Sulphate and group D was kept as untreated control (Table 2).

Mortality, postmortem findings, cumulative feed consumption, weight gain and feed conversion ratio and in each group was recorded and results were compared. Data was statistically analyzed / compared accordingly.

\section{Results}

Data collected from thirty $E$. coli infected farms showed that antibiotics in $E$. coli infection were mostly used in combination. Most frequently used combination contained enrofloxacin plus colistin (Table 3). Data also revealed that most of the farmers were totally illiterate about antibiotic usage, efficacy, storage and recommended dosage of different antibiotics (Table 4).

\section{Effect of treatment on mortality in groups}

Observations with respect to mortality are shown (Table 5). Total mortality throughout the entire experimental in all groups was 30 .

\section{Postmortem findings in groups}

Thickened and cloudy air sacs and in severe cases, caseous exudates on air sac was present. Adhesive pericarditis and fibrinous perihepatitis were present in most of the cases. Intestinal infections with enteritis with excessive mucus and diarrhea (yellowish droppings) were common.

Effect of treatment on cumulative feed consumptions, weight gain and FCR in groups

Effect of treatment on cumulative feed consumptions, weight gain and FCR in groups has been shown (Table 6-9).

\section{Discussion}

To evaluate the efficacy of conjoined therapy (enrofloxacin plus colistin) under in vivo condition, pathogenic strains of $E$. coli were selected. For identification of $E$. coli, a smear was prepared from growth on MacConkey's agar and stained with gramstaining. The isolates were cocco-bacillary, long filamentous and pinkish in color. These cultural and morphological characteristics are consistent with previous reports [17].

Table 1. Vaccine schedule of experimental chicks

\begin{tabular}{|c|c|c|c|}
\hline Age(Days) & Name of Vaccine & Shot ${\left(\mathbf{1}^{\text {st }} / \mathbf{2}^{\text {nd }}\right)}_{c \mid}^{\text {Route }}$ \\
\hline 5 & Newcastle disease (ND) & $1^{\text {st }}$ & E/D \\
\hline 10 & Infectious bursal disease (IBD) / Gumboro & $1^{\text {st }}$ & E/D \\
\hline 17 & Hydropericardium syndrome & Single & S/C \\
\hline 20 & IBD & $2^{\text {nd }}$ & D/W \\
\hline 24 & ND & $2^{\text {nd }}$ & D/W \\
\hline
\end{tabular}

E/D = Eye drop, $\mathrm{S} / \mathrm{C}=$ Sub Cutaneous, $\mathrm{D} / \mathrm{W}=$ Drinking water 
Table 2.Antibiotic treatments in different groups

\begin{tabular}{|c|c|c|c|c|c|}
\hline Group & \multicolumn{2}{|c|}{$\mathbf{A}$} & $\mathbf{B}$ & C & D \\
\hline No. of birds & \multicolumn{2}{|c|}{35} & 35 & 35 & 35 \\
\hline Quantity of inoculums & \multicolumn{2}{|c|}{$1 \mathrm{~mL}$} & $1 \mathrm{~mL}$ & $1 \mathrm{Ml}$ & $1 \mathrm{~mL}$ \\
\hline $\begin{array}{c}\text { Concentration of } \\
\text { inoculums }\end{array}$ & \multicolumn{2}{|c|}{$1 \times 10^{6} \mathrm{cfu}$} & $1 \times 10^{6} \mathrm{cfu}$ & $1 \times 10^{6} \mathrm{cfu}$ & $1 \times 10^{6} \mathrm{cfu}$ \\
\hline $\begin{array}{c}\text { Antibiotic usage } \\
\text { (combination / single) }\end{array}$ & \multicolumn{2}{|c|}{ Combination of two antibiotics } & Single & Single & Nil \\
\hline $\begin{array}{c}\text { Treatment (antibiotic } \\
\text { usage) }\end{array}$ & Enrofloxacin & $\begin{array}{l}\text { Colistin } \\
\text { sulphate }\end{array}$ & Enrofloxacin & $\begin{array}{l}\text { Colistin } \\
\text { sulphate }\end{array}$ & Nil \\
\hline Dose rate (@) & $1 \mathrm{~mL} / 4 \mathrm{~L}$ & $1 \mathrm{gm} / 10 \mathrm{~L}$ & $1 \mathrm{~mL} / 4 \mathrm{~L}$ & $1 \mathrm{gm} / 10 \mathrm{~L}$ & Nil \\
\hline Route of treatment & DW & DW & DW & DW & Nil \\
\hline Name of brand & Vet Enrox-20 & Colisol & Vet Enrox-20 & Colisol & Nil \\
\hline Name of manufacturer & $\begin{array}{c}\text { Vetycare } \\
\text { Pharma, Pak }\end{array}$ & $\begin{array}{c}\text { Vetycare } \\
\text { Pharma, Pak }\end{array}$ & $\begin{array}{c}\text { Vetycare } \\
\text { Pharma, Pak }\end{array}$ & $\begin{array}{c}\text { Vetycare } \\
\text { Pharma, Pak }\end{array}$ & - \\
\hline $\begin{array}{c}\text { Duration of treatment } \\
\text { (days) }\end{array}$ & \multicolumn{2}{|c|}{5} & 5 & 5 & - \\
\hline
\end{tabular}

Table 3.Field survey of antibiotic usage in $E$. coli associated problems on 30 farms in Faisalabad district.

\begin{tabular}{|c|c|c|c|c|c|c|}
\hline \multirow[b]{2}{*}{$\begin{array}{l}\text { No. of } \\
\text { Birds }\end{array}$} & \multicolumn{3}{|c|}{ Mortality } & \multirow[b]{2}{*}{ Brand name } & \multirow[b]{2}{*}{$\begin{array}{c}\text { Name of } \\
\text { manufacturer }\end{array}$} & \multirow{2}{*}{$\begin{array}{c}\text { Antibiotic } \\
\text { usage (single } \\
\text { / } \\
\text { combination) }\end{array}$} \\
\hline & $\begin{array}{c}\text { Pre- } \\
\text { treatment }\end{array}$ & $\begin{array}{c}\text { Post- } \\
\text { treatmen } \\
t \\
\end{array}$ & $\begin{array}{c}\text { Reduction } \\
(\%)\end{array}$ & & & \\
\hline 2000 & 50 & 15 & 2.2 & Aitquine $20 \%$ & $\begin{array}{c}\text { Azieda Therapeutica, } \\
\text { Italy }\end{array}$ & Single \\
\hline \multirow{2}{*}{4000} & \multirow{2}{*}{250} & \multirow{2}{*}{20} & \multirow{2}{*}{5.75} & Enrosol-S & $\begin{array}{l}\text { Vet. \& Agri., } \\
\text { Product, Jordan }\end{array}$ & \multirow{2}{*}{ Combination } \\
\hline & & & & Colisol & $\begin{array}{c}\text { Do Pharma } \\
\text { Netherlands }\end{array}$ & \\
\hline \multirow[t]{2}{*}{3000} & \multirow[t]{2}{*}{120} & \multirow[t]{2}{*}{18} & \multirow[t]{2}{*}{3.34} & $\begin{array}{c}\text { Vetyenrox } \\
10 \% \\
\end{array}$ & $\begin{array}{c}\text { Vetycare Pharma, } \\
\text { Pak }\end{array}$ & \multirow{2}{*}{-do- } \\
\hline & & & & Colimysin & Gellini Intl., Italy & \\
\hline 10,000 & 250 & 52 & 2 & Floxamycin & $\begin{array}{l}\text { Vaksindo Pharma, } \\
\text { Indonesia }\end{array}$ & Single \\
\hline \multirow[t]{2}{*}{3000} & \multirow[t]{2}{*}{189} & \multirow[t]{2}{*}{50} & \multirow[t]{2}{*}{4.63} & Vetyerox $10 \%$ & $\begin{array}{c}\text { Vetycare Pharma, } \\
\text { Pak }\end{array}$ & \multirow[t]{2}{*}{ Combination } \\
\hline & & & & Tribressin & Glaxo Welcome, Pak & \\
\hline \multirow{2}{*}{3000} & \multirow{2}{*}{140} & \multirow{2}{*}{25} & \multirow{2}{*}{3.83} & $\begin{array}{l}\text { Vetyenrox- } \\
20 \%\end{array}$ & $\begin{array}{l}\text { Vetycare Pharma, } \\
\text { Pak }\end{array}$ & \multirow{2}{*}{-do- } \\
\hline & & & & Colisol & $\begin{array}{c}\text { Do Pharma } \\
\text { Netherlands }\end{array}$ & \\
\hline \multirow[t]{2}{*}{4000} & \multirow[t]{2}{*}{398} & \multirow[t]{2}{*}{85} & \multirow[t]{2}{*}{7.85} & Enrosol-S & $\begin{array}{l}\text { Vet. \& and Agri. } \\
\text { Products, Jordan }\end{array}$ & \multirow[t]{2}{*}{-do- } \\
\hline & & & & Colimycin & Gellini Intl., Italy & \\
\hline \multirow{2}{*}{2500} & \multirow{2}{*}{200} & \multirow{2}{*}{120} & \multirow{2}{*}{3.2} & Vetyerox $10 \%$ & $\begin{array}{c}\text { Vetycare Pharma, } \\
\text { Pak }\end{array}$ & \multirow{2}{*}{-do- } \\
\hline & & & & Colisol & $\begin{array}{l}\text { Do Pharma } \\
\text { Netherlands }\end{array}$ & \\
\hline 3500 & 450 & 125 & 1128 & $\begin{array}{c}\text { Vetyenorx20 } \\
\% \\
\end{array}$ & $\begin{array}{c}\text { Vetycare Pharma, } \\
\text { Pak } \\
\end{array}$ & $-d o$ \\
\hline 3500 & 450 & 125 & 11.28 & Colisol & $\begin{array}{c}\text { Do Pharma } \\
\text { Netherlands }\end{array}$ & -do- \\
\hline 2200 & 290 & 108 & 8.27 & Vetyenrox $20 \%$ & $\begin{array}{c}\text { Vety-care Pharma, } \\
\text { Pak }\end{array}$ & - do- \\
\hline & & & & Colimycin & Gellini Intl., Italy & \\
\hline 2700 & 185 & 130 & 2.03 & Aitquine & $\begin{array}{l}\text { Zieda Therapentica, } \\
\text { Italy }\end{array}$ & Single \\
\hline
\end{tabular}




\begin{tabular}{|c|c|c|c|c|c|c|}
\hline \multirow[t]{2}{*}{3200} & \multirow[t]{2}{*}{300} & \multirow[t]{2}{*}{65} & \multirow[t]{2}{*}{7.34} & Enrosol-S & $\begin{array}{c}\text { Vet.\& Agri. Products, } \\
\text { Jordan }\end{array}$ & \multirow[t]{2}{*}{ Combination } \\
\hline & & & & Colimycin & Gellini Intl., Italy & \\
\hline \multirow{2}{*}{4000} & \multirow{2}{*}{311} & \multirow{2}{*}{105} & \multirow[t]{2}{*}{5.15} & Enrosol-s & $\begin{array}{l}\text { Vet. \& Agri. } \\
\text { products, Jordan }\end{array}$ & \multirow{2}{*}{-do- } \\
\hline & & & & Colisol & $\begin{array}{c}\text { Do Pharma } \\
\text { Netherlands }\end{array}$ & \\
\hline \multirow{2}{*}{3800} & \multirow{2}{*}{296} & \multirow{2}{*}{90} & \multirow{2}{*}{5.43} & $\begin{array}{l}\text { Vetyenrox } \\
10 \%\end{array}$ & $\begin{array}{c}\text { Vetycare Pharma, } \\
\text { Pak }\end{array}$ & \multirow{2}{*}{-do- } \\
\hline & & & & Colisol & $\begin{array}{c}\text { Do Pharma } \\
\text { Netherlands }\end{array}$ & \\
\hline \multirow{2}{*}{3200} & \multirow{2}{*}{320} & \multirow{2}{*}{100} & \multirow{2}{*}{6.87} & Enrosol-S & $\begin{array}{c}\text { Vet. \& Agri. } \\
\text { products, Jordan }\end{array}$ & \multirow{2}{*}{-do- } \\
\hline & & & & Colisol & $\begin{array}{l}\text { Do Pharma, } \\
\text { Netherlands }\end{array}$ & \\
\hline 2500 & 150 & 69 & 3.24 & Floxamycin & $\begin{array}{l}\text { Vaksindo Pharma } \\
\text { Indonesia }\end{array}$ & Single \\
\hline \multirow{2}{*}{3500} & \multirow{2}{*}{320} & \multirow{2}{*}{100} & \multirow{2}{*}{6.28} & $\begin{array}{c}\text { Vetyenrox } \\
10 \% \\
\end{array}$ & $\begin{array}{c}\text { Vetycare Pharma, } \\
\text { Pak }\end{array}$ & \multirow{2}{*}{ Combination } \\
\hline & & & & Colisol & $\begin{array}{c}\text { Do Pharma } \\
\text { Netherlands }\end{array}$ & \\
\hline \multirow{2}{*}{4200} & \multirow{2}{*}{435} & \multirow{2}{*}{80} & \multirow{2}{*}{8.45} & Enrosol-S & $\begin{array}{c}\text { Vet. \& Agri. } \\
\text { products, Jordan }\end{array}$ & \multirow{2}{*}{-do- } \\
\hline & & & & Colisol & $\begin{array}{c}\text { Do Pharma } \\
\text { Netherlands }\end{array}$ & \\
\hline 2000 & 158 & 80 & 3.25 & $\begin{array}{c}\text { Flumiquine- } \\
20 \%\end{array}$ & -- & Single \\
\hline 7500 & 102 & 00 & 526 & Enrosol-S & $\begin{array}{l}\text { Vet. \& Agri. } \\
\text { products, Jordan }\end{array}$ & Combingtion \\
\hline 1500 & 492 & 90 & 5.50 & Colisol & $\begin{array}{c}\text { Do Pharma } \\
\text { Netherlands }\end{array}$ & combination \\
\hline 2500 & 205 & 180 & 5.46 & $\begin{array}{c}\text { Vetyenrox } \\
10 \%\end{array}$ & $\begin{array}{l}\text { Vetycare Pharma, } \\
\text { Pak }\end{array}$ & $\mathrm{do}$ \\
\hline 2500 & 200 & 180 & 5.40 & Colisol & $\begin{array}{c}\text { Do Pharma } \\
\text { Netherlands }\end{array}$ & $-\mathrm{do}-$ \\
\hline 5500 & 450 & 125 & 500 & Vetyenrox $20 \%$ & Vetyare Pharma, Pak & do \\
\hline 5500 & 450 & 125 & 5.90 & Colimycin & Gellini Intl., Italy & -do- \\
\hline 3100 & 220 & 105 & 3.7 & Aitquine & $\begin{array}{l}\text { Zieda Therapentica, } \\
\text { Italy }\end{array}$ & Single \\
\hline 2800 & 390 & 127 & 9.39 & Tribressin & $\begin{array}{c}\text { Vet. \& Agri. } \\
\text { products, Jordan }\end{array}$ & Combination \\
\hline & & & & Enrosol-S & -do- & \\
\hline 6000 & 440 & 135 & 5.08 & Vetyerox-10\% & $\begin{array}{c}\text { Vetycare Pharma, } \\
\text { Pak }\end{array}$ & Single \\
\hline 5000 & 305 & 90 & 4.43 & Aitquine & $\begin{array}{l}\text { Zieda Therapentica, } \\
\text { Italy }\end{array}$ & -do- \\
\hline 2900 & 297 & 85 & 7.41 & $\begin{array}{c}\text { Vetyenrox } \\
10 \%\end{array}$ & $\begin{array}{l}\text { Vetycare Pharma, } \\
\text { Pak }\end{array}$ & Combination \\
\hline & & & & Colimysin & Gellini Intl., Italy & \\
\hline 2500 & 400 & 290 & 4.4 & $\begin{array}{c}\text { Vetyenrox } \\
10 \% \\
\end{array}$ & $\begin{array}{c}\text { Vetycare Pharma, } \\
\text { Pak }\end{array}$ & - do- \\
\hline 2500 & 400 & 290 & 4.4 & Colisol & $\begin{array}{c}\text { Do Pharma } \\
\text { Netherlands }\end{array}$ & -do- \\
\hline 2800 & 510 & 235 & 082 & Atiquine 20 & $\begin{array}{l}\text { Azieda Therapcutica } \\
\text { Italy }\end{array}$ & do \\
\hline 2800 & 510 & 235 & 9.82 & Colisol & $\begin{array}{c}\text { Do Pharma } \\
\text { Netherlands }\end{array}$ & -do- \\
\hline
\end{tabular}


Table 4. Awareness of farmers to antibiotic usage patterns

\begin{tabular}{|c|c|c|c|c|c|c|c|c|}
\hline \multirow{2}{*}{\multicolumn{2}{|c|}{ Parameters }} & \multicolumn{7}{|c|}{ Range of Poultry Farms } \\
\hline & & \multirow[t]{2}{*}{0} & \multirow{2}{*}{$\frac{1-5}{\sqrt{ }}$} & \multirow[t]{2}{*}{$6-10$} & \multirow[t]{2}{*}{$11-15$} & \multirow[t]{2}{*}{$16-20$} & \multirow[t]{2}{*}{$21-25$} & \multirow[t]{2}{*}{$26-30$} \\
\hline \multirow{3}{*}{$\begin{array}{l}\text { Source of } \\
\text { Antibiotic }\end{array}$} & Medical store & & & & & & & \\
\hline & Any other source & & & $\sqrt{ }$ & & & & \\
\hline & Distributer & & & & $\sqrt{ }$ & & & \\
\hline \multirow{3}{*}{$\begin{array}{l}\text { Antibiotic } \\
\text { Prescriber }\end{array}$} & Veterinarian & & & & $\sqrt{ }$ & & & \\
\hline & Distributor & & & $\sqrt{ }$ & & & & \\
\hline & Self & & $\sqrt{ }$ & & & & & \\
\hline \multicolumn{2}{|c|}{ Antibiotic dosage } & & & & $\sqrt{ }$ & & & \\
\hline \multicolumn{2}{|c|}{ Antibiotic withdrawal period } & $\sqrt{ }$ & & & & & & \\
\hline \multicolumn{2}{|c|}{ Price and antibiotic selection } & & & & & & & $\sqrt{ }$ \\
\hline \multicolumn{2}{|c|}{ Proper storage at farm* } & $\sqrt{ }$ & & & & & & \\
\hline \multicolumn{2}{|c|}{ Antibiotic ineffectiveness $* *$} & & & & & & & $\sqrt{ }$ \\
\hline \multicolumn{2}{|c|}{ Resistance to antibiotics } & & & & & & & $\sqrt{ }$ \\
\hline \multicolumn{2}{|c|}{ Irrational use of antibiotics } & & & $\sqrt{ }$ & & & & \\
\hline \multicolumn{2}{|c|}{ Support for combing antibiotics } & & & & & $\sqrt{ }$ & & \\
\hline
\end{tabular}

Table 5. Mortality in experimental groups

\begin{tabular}{|c|c|c|c|c|c|c|}
\hline \multirow{2}{*}{ Age (Days) } & \multirow{2}{*}{ DPI } & \multicolumn{3}{|c|}{ Mortality in experimental groups } & \multirow{2}{*}{ Total Mortality } \\
\cline { 3 - 6 } & & A & B & C & D & \\
\hline $27^{\text {th }}$ & $1^{\text {st }}$ & - & - & - & - & - \\
\hline $28^{\text {th }}$ & $2^{\text {nd }}$ & 1 & 4 & 3 & 5 & 13 \\
\hline $29^{\text {th }}$ & $3^{\text {rd }}$ & 1 & 3 & 2 & 3 & 9 \\
\hline $30^{\text {th }}$ & $4^{\text {th }}$ & - & 1 & 1 & 2 & 4 \\
\hline $31^{\text {st }}$ & $5^{\text {th }}$ & - & - & - & 2 & 2 \\
\hline $32^{\text {nd }}$ & $6^{\text {th }}$ & - & - & - & - & - \\
\hline $33^{\text {rd }}$ & $7^{\text {th }}$ & - & - & - & 2 & 2 \\
\hline $34^{\text {th }}$ & $8^{\text {th }}$ & - & - & - & - & - \\
\hline $35^{\text {th }}$ & $9^{\text {th }}$ & - & - & - & - & - \\
\hline $36^{\text {th }}$ & $10^{\text {th }}$ & - & - & - & - & - \\
\hline $37^{\text {th }}$ & $11^{\text {th }}$ & - & - & - & - & - \\
\hline $38^{\text {th }}$ & $12^{\text {th }}$ & - & - & - & - & - \\
\hline $39^{\text {th }}$ & $13^{\text {th }}$ & - & - & - & - & - \\
\hline $40^{\text {th }}$ & $14^{\text {th }}$ & - & - & - & - & - \\
\hline \multicolumn{2}{|r|}{ Total $=$} & 2 & 8 & 6 & 14 & 30 \\
\hline \multicolumn{2}{|l|}{ Percentage $(\%)=$} & 5.71 & 22.85 & 17.14 & 40 & 21.42 \\
\hline
\end{tabular}

Table 6. Cumulative feed consumption (Kg) igroups

\begin{tabular}{|c|c|c|c|c|}
\hline \multirow{2}{*}{ Age (Days) } & \multicolumn{4}{|c|}{ Cumulative feed consumption (Kg) in groups } \\
\cline { 2 - 5 } & $\mathbf{A}$ & $\mathbf{B}$ & $\mathbf{C}$ & D \\
\hline $25^{\text {th }}$ & 35.6 & 35.6 & 35 & 34.2 \\
\hline $30^{\text {th }}$ & 70.20 & 68.2 & 66.9 & 56.7 \\
\hline $35^{\text {th }}$ & 101.38 & 80.5 & 86.37 & 60.4 \\
\hline $40^{\text {th }}$ & 140.7 & 95.30 & 112.23 & 64.44 \\
\hline
\end{tabular}


Table 7. Mean body weight in groups

\begin{tabular}{|c|c|c|c|c|c|}
\hline \multirow{2}{*}{$\begin{array}{c}\text { Age } \\
\text { (Days) }\end{array}$} & \multicolumn{4}{|c|}{ Mean body weight (gm) in groups } & \multirow{2}{*}{ Mean } \\
\hline $25^{\text {th }}$ & 436.51 & 422.85 & 416.54 & 434.4 & 427.579 \\
\hline $30^{\text {th }}$ & 800.81 & 724.39 & 726 & 565.28 & 590.157 \\
\hline $35^{\text {th }}$ & 1200.06 & 1125.40 & 1099.79 & 863 & $863 . .43$ \\
\hline $40^{\text {th }}$ & 1645.09 & 1406.90 & 1456 & 1092.61 & 1092.4 \\
\hline Mean & 956.786 & 728.921 & 778.721 & 509.058 & -- \\
\hline
\end{tabular}

Table 8. Mean weight gain in groups

\begin{tabular}{|c|c|c|c|c|}
\hline \multirow{2}{*}{$\begin{array}{c}\text { Age } \\
\text { (Days) }\end{array}$} & $\mathbf{4}$ Mean weight gain (gm) in groups \\
\cline { 2 - 5 } & $\mathbf{A}$ & $\mathbf{B}$ & $\mathbf{C}$ & D \\
\hline $25^{\text {th }}$ & - & - & - & - \\
\hline $30^{\text {th }}$ & 11.03 & 8.61 & 10.31 & 5.23 \\
\hline $35^{\text {th }}$ & 12.9 & 12.32 & 12.88 & 13.53 \\
\hline $40^{\text {th }}$ & 13.90 & 10.42 & 12.72 & 10.93 \\
\hline
\end{tabular}

Table 9. Feed conversion ratio in groups

\begin{tabular}{|c|c|c|c|c|}
\hline \multirow{2}{*}{ Age (Days) } & \multicolumn{4}{|c|}{ FCR in groups } \\
\cline { 2 - 5 } & $\mathbf{A}$ & $\mathbf{B}$ & $\mathbf{C}$ & D \\
\hline $25^{\text {th }}$ & 2.26 & 2.33 & 2.33 & 2.18 \\
\hline $30^{\text {th }}$ & 2.49 & 2.77 & 2.93 & 2.72 \\
\hline $35^{\text {th }}$ & 2.48 & 2.55 & 2.61 & 2.79 \\
\hline $40^{\text {th }}$ & 2.45 & 2.51 & 2.65 & 2.89 \\
\hline
\end{tabular}

Biochemical reactions of $E$. coli isolates were studied. On triple sugar iron agar, the isolates fermented lactose and sucrose with production of gas, but hydrogen sulphide was not produced on Simon Citrate medium, the isolates change the color of the medium from original green to blue and colonies were streaked on the medium. Similarly, researchers studied the biochemical reaction of 267 strains of $E$. coli and found fermentation of glucose with the production of acid and gas, sucrose was fermented by 153 strains manitol by 267, salicin by 14 and dulcitol by 161 with 20 late fermenters. Methyl red was positive for 267. However, none of strains produced hydrogen sulphide [18]. All E. coli isolates are not pathogenic and is quite necessary to distinguish between pathogenic and nonpathogenic strains.

In our study the pathogenicity of $E$. coli was determined using intestinal loope ligation method. Researchers had also adopted intestinal loop ligation method for the evaluation of pathogenicity of $E$. coli [19].
In the present study intestinal loop ligation method, the positive samples (i.e pathogenic) of E. coli were identified by increased size (i.e triple fold than normal) and increased luminal fluid of the intestine. Present results also coincide with the previous studies [16].

In vivo, the disease was produced experimentally by oral administration of $1 \times 10^{6} \mathrm{cfu}$ (bacilli) / $\mathrm{mL}$ in all the groups at the age of $26^{\text {th }}$ days. The experimental disease of $E$. coli can also be produced through other routes i.e. intravenous, etc as described by different method [20, 21].

In the present study primary lesions of experimentally induced colisepticemia were pericarditis, perihepatitis and air sacculitis. Pericardial fluid became progressively more fibrinous. Necrotic foci in the heart muscles were present in the chicks which succumbed to infection. Pericardial sac was thickened and pale colored gelatinous exudates. Congestion of liver, spleen, kidney and small intestine were also observed. Similar lesions are also 
reported [22-24]. The present findings of described pathological lesions also coincide researchers who found petechiation of intestines, heart, lungs, kidneys and enlargement of liver in about $50 \%$ of the cases $[25,26]$.In our study small necrotic foci were also found in the liver. Fibirnous pericarditis, hepatitis and air sacculitis in experimentally produced $E$. coli infection is also reported [20]. The liver was markedly enlarged and covered by gelatinous material. Various forms of $E$. coli infection cause $8-17 \%$ mortality in the affected birds. Fibrinous pericarditis followed by air sacculitis, septicemia, perilobular hepatitis and polyserositis has also been reported [15].

In present study, after appearance of clinical signs of colibacillosis birds of all the groups were treated as described above. Mortality in group A was lowest (5.71\%), mortality in group B and C was 22.86 and. $17.14 \%$, respectively, while mortality in group D was highest (40\%). Least mortality was recorded in group A which was medicated with combination of enrofloxacin plus colistin sulphate. This trail showed that combination of enrofloxacin and colistin is highly effective against avian colibacillosis. FCR of broiler chicks kept in the group A, B, C and D were $2.45,2.51,2.65$ and 2.89 respectively. FCR increased markedly in group D which were infected with $E$. coli but were not treated. The increased FCR in group D might be due to the anorexia which might have caused low body weight gain.

\section{Authors' contributions}

Conceived and designed the experiments: $T$ Ahmad \& Ghulam Muhammad, Performed the experiments: T Ahmad, Analyzed the data: T Ahmad, G Muhammad, A Sharif \& A Shakoor, Contributed materials / analysis / tools: G Muhammad, A Sharif, A Shakoor, M Nadeem \& M Rizwan, Wrote the paper: T Ahmad, A Sharif, M Nadeem \& Muhammad Rizwan

\section{References}

1. Haneef W \& Siddique M (1990). Prevalence and antibiogram of
Escherichia coli isolates in and around Faisalabad. Proc. $3^{\text {rd }}$ Intl. Cong., Pak. Vet. Med. Assoc. pp: 285-292.

2. Nasralla HH \& Reda MI (1980). Colibacillosis in chicks in Saudi Arabia. Trop Anim Health Pro 12(1): 15-16.

3. Qureshi MS (1987). Poultry status in Pakistan: A report. Poultry Dev Center (PRI) Rawalpindi 21-23.

4. Bhatti BM (1989). Incidence of poultry disease and their importance in poultry production in Pakistan. Pak Vet $J$ 9:194-197.

5. Mukhopadhyaya BN \& Mishra SD (1992). Incidence of colibacillosis in chickens in some poultry pockets of west Bangal. Indian J Poult Sci 27(2): 103-107.

6. Ginns CA, Browning GF, Benhan ML, Anderson GA \& Whithear KG (1996). Antimicrobial resistance and epidemiology of Escherichia coli in broiler breeder chickens. Avian Pathol, 25(3): 591-605.

7. Tariq MA (1989). Further studies on the status of drug resistance in pathogenic avian Escherichia coli. Pak Vet J 9: 165-167.

8. Giraud E, Setrin SL, Flaujac G, Cloeckaert A, Moulin MD \& Dancla EC (2001). Characterization of highlevel fluoroquinolone resistance in Escherichia coli O78:K80 isolated from turkeys. J Antimicrob Chemoth 47(3): 341-343.

9. Hinz KH \& Will B (1988). The antibacterial in vitro and in vivo effectiveness of enrofloxacin against Haemophilus paragallinarum. Berl Munch Tierarztl 101 (12): 409-412.

10. Anadon A, Larrañaga MRM, Díaz MJ, Bringas P, Martínez MA, Cruz MLF, Fernández MC \& Fernández R (1995). Pharmacokinetics and residues of enrofloxacin in chickens. Am J Vet Res 56(4): 501-505.

11. Saleem M, Muhammad G, Siddique M \& Zia T (1999). In vitro antibiotic susceptibility profiles of avian 
Escherichia coli in and around Faisalabad. Pak Vet J 19(3): 139-141

12. Prescott JF \& Baggot JD (1993). Antimicrobial therapy in veterinary medicine, $2^{\text {nd }}$ ed., The Iowa State University Press, Ames, Iowa USA, pp: 133-136.

13. Cruickshank R (1975). Medical microbiology: the practice of medical microbiology. 12 ${ }^{\text {th }}$ Ed. The English Language Book Society and E. Livingstone, London, UK. pp: 211215.

14. Cottral EG (1978). Manual of Standardized Methods for Veterinary Microbiology Cornstock Pub. Assoc. Ithaca, USA. pp 50-56.

15. Cheesbrough M (1991). Medical laboratory manual for tropical countries. Vol. II. Tropical Health Technology, Doddington, England; Butter-worth (Publishers) Ltd., London.

16. Altwegg M \& Bochkemiki J (1998). Escherichia and Shigella In: Topeland Wilson's Microbiology and Microbial infections. Ed. 9 ${ }^{\text {th }}$, Vol.2. Arnold, London, pp: 946-947.

17. Merchant LA \& Packer RA (1971). Veterinary bacteriology and virology. $7^{\text {th }}$ Ed. The Iowa state University Press, Ames Iowa, USA. pp: 332-341.

18. Heller ED \& Perek M (1968). Pathogenic Escherichia coli strains prevalent in poultry flocks in Israel. British Vet J 124(11): 509-513.
19. Akhtar M, Ashfaqe M, Afzal H \& Aqaf M (1991). Comparison of Congo red and biochemical tests in differentiating enteropathgenic Esherichia coli. J Amin Plant Sci 1: 63-66.

20. Sajka WJ \& Carngham BA (1961). Escherichia coli infection in poultry. Res Vet Sci 2: 340-352.

21. Durant AJ \& McDougle HC (1947). Escherichia coli in the blood stream of adult fowl affected with ocular form of fowl paralysis. Am J Vet Res 8(27): 213-215.

22. Gross WB (1957). Escherichia coli infection of the chicken eye. Avian Dis 1(1): 36-41.

23. Gross WB (1962). Blood cultures, blood counts and temperature records in an experimentally produced "air sac disease" and uncomplicated Escherichia coli infection of chickens. Poultry Sci 41(3): 691-700.

24. Verma KC \& Adlakha SC (1971). Escherichia coli and other enterobacteriace group of organisms from different pathological conditions on poultry. Indian Vet J48: 107-108.

25. Nagi MS \& Khanna PN (1967). A cholera like disease in chicken due to hemolytic E. coli. Indian Vet J 44(8): 629-633.

26. Belishkil B \& Panikat II (1969). Pathogenicity of $E$. coli isolated from broiler with septicemia. Vet Moscow 12: 25-26. 